\title{
Être un enseignant débutant dans un système éducatif traversé par multiples référentiels
}

Le cas du Rwanda

Being a beginning teacher in an education system traversed by multiple frames

of reference. The case of Rwanda

Ser un profesor principiante en un sistema educativo atravesado por referentes múltiples. El caso de Ruanda

\section{Masengesho Kamuzinzi}

\section{OpenEdition}

\section{Journals}

Édition électronique

URL : https://journals.openedition.org/ries/5792

DOI : 10.4000/ries.5792

ISSN : 2261-4265

\section{Éditeur}

France Education international

\section{Édition imprimée}

Date de publication : 1 avril 2017

Pagination : 47-55

ISBN : 978-2-85420-614-2

ISSN : $1254-4590$

Référence électronique

Masengesho Kamuzinzi, «Être un enseignant débutant dans un système éducatif traversé par multiples référentiels », Revue internationale d'éducation de Sèvres [En ligne], 74 | avril 2017, mis en ligne le 01 avril 2019, consulté le 11 mars 2022. URL : http://journals.openedition.org/ries/5792 ; DOI : https://doi.org/10.4000/ries.5792 


\section{Être un enseignant débutant dans un système éducatif traversé par multiples référentiels}

\section{Le cas du Rwanda}

\section{Masengesho Kamuzinzi \\ Université du Rwanda}

Cet article porte sur l'expérience des enseignants du niveau primaire au Rwanda, dans un système éducatif traversé par plusieurs référentiels, témoins des multiples influences qu'il a subies depuis l'instauration de l'école moderne en 1900 .

\section{UN SYSTÈME AUX MULTIPLES RÉFÉRENCES}

Les systèmes éducatifs des États de l'Afrique subsaharienne sont classés en trois blocs dominants, en fonction de leur affiliation au modèle anglo-saxon, au modèle " francophone » ou au modèle portugais. Les systèmes affiliés au modèle anglo-saxon sont, entre autres, caractérisés par une faible régulation étatique ayant pour corollaire l'éclatement de ces systèmes en écoles multi-niveaux aux traits caractéristiques très différents. On y remarque également un goût prononcé pour l'initiative privée, avec comme conséquence une grande marge de manœuvre dans la création de nouveaux types d'écoles et de nouveaux curricula. En bref, ces systèmes sont caractérisés par une grande hétérogénéité, à cause de la faible standardisation des programmes enseignés dans les différents réseaux.

L'autre modèle dominant inclut les systèmes éducatifs des anciennes colonies françaises. En y regardant de près, on remarque que ces systèmes inspirés du modèle français partagent plusieurs traits avec ceux des anciennes colonies belges de la région des grands lacs. Cet ensemble, que l'on peut réunir sous le label de "modèle francophone ", est surtout caractérisé par l'omniprésence de l'État régulateur et par la forte imprégnation de la philosophie de "l'école républicaine" inspirée du modèle français. Ces systèmes fonctionnent surtout sur la base des mêmes standards, alors que les référentiels qui sous-tendent les systèmes éducatifs inspirés du modèle anglo-saxon sont assez éclatés. En outre, dans la plupart de ces systèmes, les politiques éducatives et les programmes d'enseignement transcendent les clivages laïc/confessionnel et public/privé. Les caractéristiques du modèle portugais ne sont pas décrites dans cet article car il n'a pas eu d'influence significative sur le système éducatif rwandais. 
Depuis l'avènement de l'école moderne avec la colonisation allemande suivie de la tutelle belge jusqu'en 1994, beaucoup de traits caractéristiques alignaient le système éducatif rwandais plutôt sur le modèle "francophone ». Jusqu'en 1980, l'organisation du système éducatif revenait exclusivement à l'État. Hormis les petits séminaires catholiques, aucune autre initiative privée n'était tolérée. Le contrôle excessif de l'État a eu, entre autres, comme effet l'homogénéisation du système. Même après l'introduction de l'initiative privée à partir de 1982, le programme national, dont la définition relevait exclusivement du ressort du gouvernement central, s'imposait à tous les réseaux et types d'écoles. Dans ce contexte, la formation des enseignants du primaire visait essentiellement à les initier à transmettre efficacement les éléments de ce programme officiel.

Mais depuis 1994, on remarque que le système éducatif rwandais oscille entre le modèle anglo-saxon et le modèle dit "francophone ", sans parvenir à s'ancrer dans l'un des deux ou tout au moins à accepter son caractère hybride, quitte à y adapter ses politiques éducatives et ses curricula ! L'affiliation au modèle anglo-saxon se remarque notamment dans l'extrême diversification de l'offre éducative à tous les niveaux. Des écoles aux philosophies très différentes et suivant parfois des curricula très différents cohabitent à l'intérieur d'un même réseau. Mais l'omniprésence de la régulation étatique inspirée de l'ancien modèle n'a pas pour autant disparu. Ainsi, par exemple, les élèves évoluant dans ces différents réseaux et types d'écoles, qui ne suivent pas nécessairement les mêmes programmes, sont tous soumis au même concours national de passage au secondaire. Comment les enseignants débutants ressentent-ils leurs premières expériences dans un système traversé par autant de référentiels?

\section{LE « CHOC ॥ DU PREMIER CONTACT AVEC LA SITUATION DE CLASSE}

Depuis les années soixante, les difficultés rencontrées par les enseignants débutants ont fait l'objet d'une abondante littérature dans toutes les langues. Par exemple, Veenman (1984) a répertorié jusqu'à 83 articles publiés en anglais sur les expériences des nouveaux arrivants dans le métier. Depuis cette période, l'intérêt des chercheurs pour l'expérience des enseignants en début de carrière n'a cessé de croître et de s'orienter dans plusieurs directions. Si les recherches anciennes se sont le plus attardées sur des variables objectives liées directement à la complexité de la tâche d'enseignement/apprentissage, depuis les années quatre-vingt-dix, les chercheurs se sont de plus en plus tournés vers des aspects touchant à la dimension «subjective » de l'expérience. En guise d'illustration, on peut citer la recherche de Pajares et Urdan (2006) sur le rôle des valeurs et croyances personnelles sur l'efficacité professionnelle des nouveaux arrivants, celle de Van Veen et Sleegers (2009) sur la médiation des émotions dans la perception de la pertinence des nouvelles réformes, celle de Ria et Durand (2001) sur la tonalité émotionnelle des premières expériences en classe, etc. 
Une des conclusions les plus récurrentes dans ces études est que le passage de l'idéal de la formation à la réalité de classe produit souvent un choc chez les nouveaux arrivants. Et ce phénomène s'observe partout dans le monde, bien que les facteurs à la base de leur frustration diffèrent d'un système à l'autre. L'universalité du phénomène s'observe à travers les termes utilisés dans différentes langues pour exprimer ce choc de premier contact avec les élèves. Dans de nombreux textes en français, on parle surtout de choc de la première expérience en classe (Martineau et Presseau, 2003). Dans la littérature anglo-saxonne, ce premier contact avec la complexité de la tâche est souvent mentionné à travers différentes anecdotes comme un "reality schock», " transition schock» ou "praxis schock». Dans la littérature germanophone, ce premier choc est désigné parfois par le vocable «Reinwascheffekt»(Veenman, 1984).

Bref, dans tous les systèmes éducatifs du monde moderne, le premier contact avec la situation de classe représente un moment de vérité pour les enseignants débutants. Ils comprennent soudain que la conception idéaliste du métier entretenue par les écoles de formation ne correspond que trop peu à la complexité de la tâche. Sur l'échantillon de cinquante enseignants en début de carrière qui ont été interrogés au cours d'une enquête de terrain, trente-huit enseignants, soit $76 \%$, ont reconnu avoir ressenti un choc au premier contact avec la situation de classe. Bien que la plupart des difficultés mentionnées par ces enseignants aient été observés dans d'autres systèmes, leur mise en contexte est nécessaire pour bien comprendre la façon dont elles se posent dans le système éducatif rwandais. Dans le point suivant, ces difficultés sont discutées en rapport avec les trois axes du triangle didactique tels que définis par Houssaye et Hameline (1992).

\section{LES ENSEIGNANTS DÉBUTANTS EN SITUATION DE CLASSE}

Plus concrètement, nous nous intéressons à la manière dont les enseignants débutants se positionnent dans la triple relation : " maître-savoirs " (transposition didactique) ; "maître-élèves » (relation pédagogique) et "élèvessavoirs»(relation d'apprentissage).

\section{La relation " maître-savoir " dans un système aux référentiels conflictuels}

Les défis identifiés par les enseignants débutants font surtout mention de difficultés de planification des leçons, en particulier la transposition didactique des savoirs savants en savoirs effectivement enseignés. Dans les études antérieures, le défi lié à la planification a été surtout discuté en rapport avec le découpage du curriculum en leçons enseignables et leur programmation à court et moyen terme. Dans la conception traditionnelle du curriculum, ce découpage est guidé par la logique de succession des matières à enseigner. Ici, la difficulté 
des nouveaux enseignants tient surtout à l'identification des prérequis et des matières qui doivent suivre. Dans la nouvelle conception inspirée de l'approche par compétences, la programmation et l'évaluation des leçons est plutôt guidée par le principe d'intégration des matières (De Ketele et Gérard, 2005). Ici, la difficulté des nouveaux enseignants porte sur l'élaboration d'une programmation intégrant des unités venant de différentes branches, pour s'assurer que ces dernières concourent à la construction de socles des compétences plus élaborés.

L'un des traits caractéristiques montrant que le système éducatif rwandais est traversé par des référentiels conflictuels est que ces deux logiques cohabitent dans le système. En examinant les différentes réformes initiées au cours de la dernière décennie, on remarque qu'à partir de 2007, le ministère de l'éducation a choisi de réduire le nombre d'intitulés figurant au programme, pour, disait-on, intégrer dans les mêmes cours les connaissances nécessaires pour une éducation de base de qualité pour tous. Par la suite, le programme de formation des enseignants du primaire s'y est adapté et s'est spécialisé par branche d'enseignement. Alors que dans l'ancien système, les enseignants du primaire étaient formés pour enseigner tous les cours figurant au programme, actuellement les nouveaux enseignants sont "spécialisés " (dans l'enseignement des langues, des mathématiques, des sciences et technologies élémentaires ou des études sociales).

Mais, à partir de 2015, les programmes enseignés au niveau primaire ont été revus à nouveau pour mieux les adapter au principe d'intégration des compétences. En l'état actuel du système, on se retrouve donc avec des enseignants formés dans un modèle (spécialisation par branche d'enseignement) mais auxquels la nouvelle réforme demande d'intégrer les matières venant de différentes branches (dans lesquelles les nouveaux enseignants n'ont pas été formés de façon certaine) pour produire des socles communs de compétences adaptées à la réalité de la vie ! Dans un tel contexte marqué par des référentiels conflictuels, comment s'y prennent les enseignants débutants?

Les observations de terrain font ressortir trois types de stratégies. Elles dépendent surtout du style d'encadrement mis en place par la direction de l'école. Dans les écoles où le système d'encadrement est plus participatif, le travail de programmation est fait de manière concertée par tous les enseignants. Ici, les nouveaux venus apprennent à partir de l'expérience des anciens et reçoivent des conseils au cas par cas. Certaines écoles tentent même de respecter le principe d'intégration des compétences, par exemple en programmant concomitamment des chapitres issus de différentes branches qui traitent des thèmes similaires et sont donc susceptibles de contribuer à la construction des compétences plus élaborées. Mais les effets escomptés d'une telle initiative sont quelque peu amoindris par le fait que ces unités de matières sont dispensées par des enseignants différents. Comme chaque enseignant ne maîtrise que ce qui se passe dans son propre cours, aucun ne peut s'assurer que ce que font les autres enseignants dans leurs propres cours concourt effectivement à la construction d'un socle commun de compétences. 
Dans la deuxième catégorie d'écoles, la programmation classique basée sur une sorte de succession "linéaire» des leçons est toujours prédominante. Dans certains cas, les enseignants ne prennent même pas le temps d'examiner le programme de manière approfondie pour identifier les prérequis et les matières qui vont suivre. La plupart d'entre eux perçoivent l'exercice de la programmation des leçons plus comme une exigence administrative que comme une préparation en amont des leçons futures. Compte tenu de l'influence de l'approche par compétence, dans ce genre d'écoles où le style d'encadrement est essentiellement bureaucratique, aucune réflexion sérieuse relative à l'intégration des matières n'accompagne cet exercice. Dans ce contexte, les anciens enseignants ont tendance à répliquer les programmations utilisées au cours des années passées, sans nécessairement les mettre en contexte. Les nouveaux arrivants n'apprennent pas non plus grand-chose de la part des anciens. À la moindre demande d'aide, les anciens se contentent de leur prêter les canevas de répartition des matières des années précédentes, sans prendre le temps de leur expliquer les tenants et les aboutissants d'une telle programmation! Dans la troisième catégorie d'écoles, aucune forme d'aide ni de référence antérieure n'est proposée aux nouveaux enseignants.

En ce qui concerne la transposition des connaissances savantes en connaissances enseignées, la spécificité du système éducatif rwandais tient surtout à la médiation de plusieurs langues dans l'organisation de la cognition. Dans l'ancien système, toutes les matières étaient enseignées dans la langue maternelle. Le français occupait une position assez marginale car il ne figurait sur l'horaire que comme un cours parmi tant d'autres. Et, comme au niveau primaire, tous les curricula et manuels scolaires étaient rédigés en kinyarwanda, la langue dans laquelle les enseignants avaient été eux-mêmes socialisés, la transposition didactique s'opérait plutôt plus facilement, car tout se passait dans la langue maternelle. Dans l'état actuel du système, les trois langues sont en compétition. Dans le réseau privé, certaines écoles commencent par enseigner exclusivement en français ou exclusivement en anglais, puis se mettent au trilinguisme après que la langue de premier choix a été maîtrisée. Même si le réseau public a récemment réintroduit la langue maternelle comme médium d'enseignement au premier cycle du primaire, dans la pratique, les enseignants passent fréquemment du kinyarwanda au français et du français à l'anglais en fonction des circonstances, comme s'ils nageaient au gré des vagues! Comment s'opère la transposition didactique dans un tel contexte multilingue?

La stratégie mise en place par les enseignants rwandais dans l'usage de plusieurs langues, par ailleurs souvent mal maîtrisées, est pour le moins originale. Nous avons par exemple remarqué que les nouveaux enseignants conceptualisent d'abord la matière à enseigner dans la langue dans laquelle ils l'ont eux-mêmes apprise et la convertissent ensuite en anglais ou en français en fonction de l'affiliation de leur école. Au lieu de se référer aux curricula et aux manuels actuels, beaucoup opèrent une sorte de retour au passé et réinterprètent le matériau à leur disposition en fonction de leurs propres acquis. Nous y reviendrons plus en 
détail lorsque nous allons aborder la troisième dimension du triangle didactique : la relation " élèves-savoirs ». Hormis la médiation de la langue dans la traduction des savoirs savants en savoirs effectivement enseignées, les enseignants débutants citent également la pression d'une charge horaire excessive, avec pour corollaire la difficulté de recourir aux méthodes actives, de prendre en compte les différences individuelles et d'évaluer les acquis des élèves dans des classes aux effectifs pléthoriques.

\section{La relation maître/élèves : la spécialisation a-t-elle radicalement changé la donne?}

Pendant l'époque coloniale, l'enseignant symbolisait en quelque sorte l'entrée dans la modernité. Ce fut le premier métier accessible aux peuples colonisés qu'on appelait alors les « indigènes ». Dans de nombreux villages, le maître symbolisait l'entrée dans la nouvelle ère, car il était le seul à s'habiller comme les colons et à parler la langue des colons. Cette image du maître-modèle a façonné la relation maître-élève, en érigeant l'enseignant en étalon de référence, l'élève occupant plutôt le statut de "disciple». Pour défendre ce statut, l'enseignant devait faire don de soi et s'assurer qu'il donnait le maximum de tout ce qu'il possédait pour préparer les nouvelles générations à embrasser cette nouvelle ère. Et, comme un seul maître dispensait tous les cours figurant au programme de sa classe, il pouvait identifier plus facilement les difficultés des élèves dans tous les domaines. Bien que l'évolution des sociétés postcoloniales ait progressivement relégué l'enseignement au rang des métiers les moins payés et donc les moins attractifs, l'image du maître-modèle n'a pas totalement disparu.

Mais la «spécialisation » a introduit des changements substantiels dans la relation "maître-élèves ». En effet, la distribution des cours par domaine de spécialité favorise l'anonymat. Chaque enseignant ne maîtrise que ce qui se passe dans son cours. Il ne peut ni se faire une idée claire des problèmes spécifiques que rencontre chaque élève ni élaborer une stratégie de récupération des élèves en difficulté car il passe trop peu de temps dans chaque classe. D’autres facteurs liés au contexte de promotion de l'éducation pour tous rendent la relation élèvesmaître encore plus problématique. Alors que, dans l'ancien système, le titulaire de la classe était le seul à juger que le niveau de l'élève était suffisant pour passer d'une classe à l'autre, dans le système de promotion de l'éducation pour tous, le taux de redoublement acceptable est fixé à l'avance et ne devrait en principe pas dépasser $10 \%$. Sur le plan pédagogique, un taux de réussite effectif à $90 \%$ est tout à fait atteignable, si l'enseignant est en charge d'une classe d'élèves motivés et dont le niveau est assez homogène. En outre, il doit bénéficier d'une collaboration satisfaisante de la part de ses collègues, des parents et de la direction. 
Dans les faits, ces conditions idéales sont loin d'être atteintes. Des dysfonctionnements sont surtout observés dans les écoles d'éducation pour tous où le droit à l'accès prime sur les autres aspects de l'encadrement. Ici, même les élèves dont les parents ne font aucun effort de collaboration ou qui abandonnent l'école sont ramenés dans l'établissement avec l'aide des autorités locales. Dans les zones où ces " abandons-retours " sont élevés, les enseignants se retrouvent avec des élèves aux niveaux disparates et dont l'implication dans l'apprentissage est très variable.

\section{La relation élèves-savoirs dans un contexte multilingue}

Les comportements des enseignants en rapport avec la transposition didactique discutée précédemment sont aussi observés chez les élèves en situation d'apprentissage. Comme nous l'avons dit, la première langue dans laquelle nous avons appris à structurer notre cognition est déterminante dans l'acquisition des nouvelles connaissances. Dans ces conditions, comment des élèves baignant au quotidien dans un multilinguisme souvent mal maîtrisé développent-ils leur relation au savoir? Le constat majeur tiré des observations de terrain est que, lorsque les enfants sont en contact avec la matière, ils la pensent d'abord en kinyarwanda, puis, une fois qu'ils ont trouvé la voie, ils convertissent leurs pensées dans l'autre langue dans laquelle il leur est demandé de réfléchir. Cette façon de faire s'observe également lors du passage d'une langue étrangère à une autre. On remarque, par exemple, que les enfants venant des familles francophones qui utilisent couramment le français à la maison réfléchissent d'abord dans cette langue et convertissent ensuite leurs trouvailles en anglais, lorsqu'ils commencent à étudier dans cette langue. Le même comportement se remarque chez les enfants issus des familles anglophones, lorsqu'ils se mettent au français.

Bref, pour les élèves comme pour les enseignants, la langue dans laquelle ils ont été socialisés pendant la prime enfance sert de référence dans l'organisation de leur cognition, qui est par la suite traduite dans d'autres langues. Il en résulte que les élèves maîtrisent mieux la matière dans la langue dans laquelle ils ont été socialisés que dans la langue officielle d'enseignement. C'est quand l'on a atteint un niveau assez élevé de maîtrise de la nouvelle langue et qu'on l'utilise au quotidien en dehors du contexte d'apprentissage que l'on commence à réfléchir à partir de cette langue, indépendamment des autres langues.

\section{LA MÉDIATION DES CROYANCES PERSONNELLES DANS LA PERCEPTION DE LA CARRIÈRE D'ENSEIGNEMENT}

La triple relation « enseignant-savoirs-élèves » a été analysée en référence à des variables objectives induites par les récentes réformes. Mais la manière dont les enseignants débutants mettent en pratique ces réformes dépend finalement 
des croyances personnelles sur leur pertinence. Une contradiction entre les convictions personnelles et les exigences des réformes induit une dissonance cognitive qui peut être source de conflit et de démotivation. Dans l'état actuel du système, la source fondamentale de dissonance chez les débutants a surtout trait à la cohabitation de deux référentiels aux principes assez opposés à l'intérieur d'un même système : le désir de former une élite capable de s'adapter dans un monde globalisé et le désir d'offrir la chance d'accès à tous.

Le premier référentiel est basé sur l'idée que, dans un monde où la concurrence est féroce, il faut préparer les nouvelles générations à la compétitivité. Ayant été formés dans cette philosophie aux accents élitistes, les enseignants débutants espèrent trouver en classe des élèves prêts à apprendre! Mais les choses se présentent différemment, surtout dans les écoles d'éducation pour tous. L'état d'esprit qui y règne est plutôt que l'accès à l'éducation de base est un droit dont aucun enfant en âge scolaire ne devrait être exclu. Dans la pratique, l'obligation de ne pas rejeter les élèves en difficulté à l'extérieur du système prime sur les exigences de qualité. Lorsque les nouveaux enseignants sont obligés de fonctionner sur la base de ce second référentiel, ils ressentent une dissonance entre leurs représentations sur l'idéal éducatif et la réalité de la classe. Pour beaucoup d'entre eux, c'est comme si les responsabilités étaient en quelque sorte inversées. Alors qu'autrefois, les efforts pour réussir étaient attendus du côté de l'élève, aujourd'hui, c'est l'enseignant qui doit se battre pour faire aimer l'école aux enfants. La plupart des enseignants débutants se sentent désarmés lorsqu'ils font face à des élèves démotivés qui ont compris que, même sans efforts, ils vont passer dans la classe supérieure compte tenu du pourcentage de redoublements autorisés.

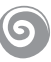

La dissonance entre leurs représentations et les exigences du système, associée aux difficiles conditions de travail (double vacation, classes pléthoriques, faible maîtrise des langues d'enseignements, salaires très bas, etc.) fait qu'à peine engagés, beaucoup d'enseignants projettent aussitôt de quitter la carrière si une opportunité se présente. La plupart d'entre eux s'accrochent à leurs postes pour trouver les moyens de payer des études universitaires (du soir ou en week-end). Mais, même dans les universités ayant des facultés d'éducation, beaucoup d'enseignants s'inscrivent dans des filières qui n'ont rien à voir avec la continuité de leur carrière. 


\section{BIBLIOGRAPHIE}

DE KETELE J.-M., GERARD F.-M. (2005) : «La validation des épreuves d'évaluation selon l'approche par les compétences ", Mesure et évaluation en éducation, 28(3), p. 1-26.

HOUSSAYE J. (1992) : Théorie et pratique de l'éducation (coll. Exploration : "Pédagogie: Histoire et Pensée), 1. Le triangle pédagogique, préface de Daniel Hameline, Berne : Peter Lang.

MARTINEAU S., PRESSEAU A. (2003) : «Le sentiment d'incompétence pédagogique des enseignants en début de carrière et le soutien à l'insertion professionnelle ", Brock Education: A Journal of Educational Research and Practice, 12(2).

PAJARES F., URDAN T. C. (2006) : Self-efficacy beliefs of adolescents, Greenwich, Connecticut : IAP.

RIA L., DURAND M. (2001) : "Les préoccupations et la tonalité émotionnelle des enseignants débutants lors de leurs premières expériences en classe », Les dossiers des sciences de l'éducation, (5), p. 111-123.

VAN VEEN K., SLEEGERS P. (2009) : "Teachers' emotions in a context of reforms: To a deeper understanding of teachers and reforms", dans Advances in teacher emotion research, p. 233-251, Springer US.

VEENMAN S. (1984) : Perceived problems of beginning teachers, Review of educational research, 54(2), p. 143-178. 
